\title{
Technique d'extraction de 12 benzodiazépines dans le plasma. Résultats expérimentaux multicentriques
}

\section{Extraction procedure of 12 benzodiazepines from plasma. Results of a multicentric study}

Nicole JOURDIL ${ }^{(1) *}$, Bernard CAPOLAGHI ${ }^{(2)}$, Michèle ARTUR ${ }^{(3)}$, Raphaël DENOOZ ${ }^{(4)}$, Nicole HOUDRET ${ }^{(5)}$, Marie-France KERGUERIS ${ }^{(\oplus)}$, Denis LAMIABLE ${ }^{()}$, Sabine COHEN ${ }^{(8)}$, Mustapha MOULSMA ${ }^{(9)}$, Nouredine SADEG ${ }^{(10)}$, Alain TURCANT ${ }^{(1)}$ (Groupe de travail "Toxicologie hospitalière" de la SFTA)

(1) UF Pharmacologie-Département de Biologie et Pathologie de la Cellule, CHU Grenoble

(2) Laboratoire de Biologie, $\mathrm{CH}$ Thionville

(3) Laboratoire de Pharmacologie et Toxicologie, CHU Dijon

(4) Laboratoire Toxi-Clinique, CHU Liège

(5) Laboratoire de Biochimie, CHU Lille

(6) Laboratoire de Pharmacologie-Toxicologie, CHU Nantes

(7) Laboratoire de Pharmacologie-Toxicologie, CHU Reims (8) Laboratoire des Urgences Biochimiques et Toxicologiques, CHU Lyon-Sud (9) UF Pharmaco-Toxicologie et Analyses des éléments trace, CHU Lyon (10) Laboratoire Claude Bernard, CH Pontoise (11) Laboratoire de Pharmacologie, CHU Angers

*Auteur à qui adresser la correspondance : Nicole JOURDIL, UF Pharmacologie-Département de Biologie et

Pathologie de la Cellule, CHU de Grenoble, Hôpital Michallon, 38043 GRENOBLE Cedex 09 Tél : 0476765492 - Fax : 0476768938 - E-mail : Njourdil@chu-grenoble.fr 


\section{RÉSUMÉ}

Dans le cadre des différents thèmes de travail proposés aux membres de la commission de Toxicologie Clinique de la SFTA, une étude est réalisée sur l'identification et la quantification simultanée de 12 benzodiazépines dans le plasma. L'objectif est de tester la robustesse d'une technique d'extraction en l'imposant aux différents participants, les conditions chromatographiques de séparation et le mode de détection restant libres, fonctions de l'équipement de chaque laboratoire. L'étude a été réalisée sur une période de 2 mois et demi. Deux échantillons tests de plasmas surchargés avec 12 benzodiazépines à des concentrations thérapeutiques ou supra-thérapeutiques sont adressés à chaque centre d'investigation en vue de leur évaluation. La technique d'extraction utilise un mélange d'hexane / dichlorométhane en milieu tamponné (pH 9.2) et le loflazépate d'éthyle comme standard interne. Une méthode CLHP avec détecteur à barrette de diode est proposée à défaut par le laboratoire coordonnateur aux participants ne disposant pas de technique d'analyse. Sur les 11 participants, 10 utilisent une technique CLHP, un seul une technique CPG. Parmi les techniques CLHP, 2 d'entre elles associent un détecteur de masse, 7 un détecteur à barrette de diodes, 1 un détecteur UV. La technique CPG est associée à un détecteur de masse. L'analyse des résultats montre que la technique d'extraction proposée a été testée avec succès par 8/11 des participants : 4/11 laboratoires sans erreur et 4/11 laboratoires avec 1 ou 2 résultats exclus. Par contre, 3/11 laboratoires ont rencontré des difficultés majeures : un site par la survenue d'émulsions lors du traitement des échantillons tests (plasmas lyophilisés à reconstituer), un site par manque de temps et d'expérience, le troisième mettant en cause le choix du standard interne en technique CPG, le loflazépate d'éthyle n'ayant pas fait l'objet d'une étude préalable par cette technique.

\section{MOTS-CLÉS}

Benzodiazépines, procédure d'extraction, identification, quantification, CL-DAD, CL-SM, CL-SM/SM, CG-SM.

\section{Introduction}

Dans le cadre des exercices menés par le groupe de travail "Toxicologie hospitalière" de la SFTA, le test de l'efficacité d'une technique d'extraction simple des benzodiazépines dans le sang, indépendamment de la technique d'analyse, est proposée à ses différents membres. Deux échantillons tests, contenant chacun plusieurs benzodiazépines à des concentrations thérapeutiques ou supra-thérapeutiques, sont transmis aux 11 laboratoires participants en vue de leur évaluation. L'objectif est de tester la robustesse d'une technique d'extraction des benzodiazépines en l'imposant à tous les participants. Il s'agit de tester la capacité d'une méthode analytique à rendre des résultats satisfaisants en présence de modifications de différentes conditions expérimentales ou environnementales (1), les conditions chromatographiques de séparation et le mode de détection restant libres, fonction de l'équipement de

\section{SUMMARY}

Within the framework of the various topics proposed to the SFTA members, a study was conducted on the simultaneous identification and quantitation of benzodiazepines in the plasma. The purpose was to test the reliability of a particular extraction procedure by imposing it to the various participants, the chromatographic conditions of separation and the detection modes remaining free, depending on the equipment available in each laboratory. The study extended over a period of two and a half months. Two plasma specimens containing overloads of twelve benzodiazepines at therapeutic and supra-therapeutic levels were supplied for evaluation to each investigation center. The extraction procedure used a mixture of hexan / dichloromethan with the use of a pH 9.2 buffer and ethyl loflazepate as internal standard. By default a HPLC method was proposed by the coordinating laboratory to the participants who didn't have any methodology. Out of 11 participants, 10 used a HPLC method, only 1 used a $G C$ method. Among the HPLC users, 2 used a mass spectrometry detector, 7 a photodiode array detector, 1 an UV detector. The GC method worked with a mass spectrometry detector. The analysis of the results showed that the extraction procedure had been successfully tested by $8 / 11$ of the participants: 4/11 have returned results without any mistakes, 4/11 have returned 1 or 2 results that had to be excluded, 3/11 have encountered major difficulties: In one laboratory emulsions occurred while processing the specimens (lyophilised plasma to be reconstituted). One laboratory lacked time and experience. The last one questioned the internal standard choice in the GC method since ethyl loflazepate hadn't been tested beforehand by this GC method.

\section{KEY-WORDS}

Benzodiazepines, extraction procedure, identification, quantification, LC-DAD, LC-MS, LC-MS/MS, GC-MS.

chaque participant. La technique d'extraction choisie, de type liquide / liquide, est volontairement simple et rapide de mise en œuvre. C'est le type de détecteur utilisé qui détermine ensuite le degré de sensibilité et de spécificité de l'analyse, limitant son application à une aide au diagnostic d'intoxication (détecteur UV ou à barrette de diodes), ou bien permettant son ouverture à de plus basses concentrations dans le cadre de la soumission chimique (détecteurs de masse). La coordination et l'exploitation des résultats de l'étude sont effectués par l'UF Pharmacologie-Département de Biologie et Pathologie de la Cellule du CHU de Grenoble.

\section{Matériel et Méthodes \\ Délai de l'étude, fourniture des échan- tillons tests et standards :}

Le délai de l'étude est fixé à deux mois et demi. Deux 
échantillons tests de plasmas contenant des surcharges en benzodiazépines à des concentrations thérapeutiques ou supra-thérapeutiques sont fournis à chaque centre d'investigation en vue de leur évaluation. Les molécules impliquées sont pour l'échantillon test 1 : le diazépam, le nordiazépam, le lorazépam, l'oxazépam, le bromazépam, le flunitrazépam, le 7-aminoflunitrazépam et pour l'échantillon test 2 : l'alprazolam, le clobazam, le midazolam, le tétrazépam et le clonazépam. Des solutions standards à $10 \mathrm{mg} / \mathrm{L}$ des différentes benzodiazépines concernées sont également fournies aux différents participants en vue de l'étalonnage des appareils.

\section{Technique de séparation et de détection proposées par défaut :}

Pour les participants ne disposant pas d'une méthode de séparation et de détection des benzodiazépines, une technique par défaut est proposée et décrite dans le Tableau I. Il s'agit d'une technique séparative CLHP couplée à un détecteur à barrette de diodes de marque Shimadzu type SPD M10 AVP. Les performances de cette technique de référence couplée à la méthode d'extraction testée sont détaillées dans le Tableau II. Pour les 12 benzodiazépines de l'étude, les linéarités sont comprises entre 0,03 et $0,30 \mathrm{mg} / \mathrm{L}$ (limites basse et haute de quantification), les répétabilités $<7,7 \%$, les reproductibilités $<12,9 \%$, les rendements d'extraction compris entre 67 et $101 \%$ et les seuils de détection compris entre 0,01 et $0,02 \mathrm{mg} / \mathrm{L}$.
Tableau I : Technique séparative et d'analyse proposée par défaut.

\begin{tabular}{|l|l|}
\hline Technique & CLHP \\
\hline Type & Barrette de diode \\
\hline Conditions chromatographiques : & \\
\hline $\begin{array}{c}\text { Caractéristiques de la colonne de } \\
\text { séparation : }\end{array}$ & \\
\hline \multicolumn{1}{|c|}{ Type de phase } & Nucléosil C18 \\
\hline Dimension ( longueur x DI) & 150 x $4.6 \mathrm{~mm}$ \\
\hline Granulométrie & $5 \mu \mathrm{m}$ \\
\hline Fabricant & Machery-Nagel \\
\hline Référence & Cat N $\mathrm{N}^{\circ}$ 720 12046 \\
\hline Température colonne & ambiante \\
\hline Volume de la boucle d'injection & $20 \mu \mathrm{l}$ \\
\hline Nature et composition de la phase mobile : & \\
\hline Phase A & Tampon phosphate $0.025 \mathrm{M}, \mathrm{pH}=2.6$ \\
\hline Phase B & Acétonitrile / eau $(90 / 10, \mathrm{v} / \mathrm{v})$ \\
\hline Proportion relative & A/B $: 85 / 15$ à T 0 \\
\hline Gradient & T $0 \mathrm{mn}:$ A/B $: 85 / 15$ \\
& T $2 \mathrm{mn}:$ A/B $: 85 / 15$ \\
& T $42 \mathrm{mn}: \mathrm{A} / \mathrm{B}: 25 / 75$ \\
& T $48 \mathrm{mn}: \mathrm{A} / \mathrm{B}: 10 / 90$ \\
& T $49 \mathrm{mn}: \mathrm{A} / \mathrm{B}: 85 / 15$ \\
& T $50 \mathrm{mn}:$ Stop \\
\hline Débit de la phase mobile & $1.3 \mathrm{ml} / \mathrm{mn}$ \\
\hline
\end{tabular}

\section{Réactifs :}

L'hexane et le dichlorométhane doivent présenter un haut degré de pureté compatible avec l'analyse chromatographique. Le tampon carbonate $\mathrm{pH} 9,2$ est préparé à partir d'un mélange de carbonate acide de sodium $(1,68 \mathrm{~g} / 100 \mathrm{~mL}$ d'eau) (a) et de carbonate de sodium $(2,12 \mathrm{~g} / 100 \mathrm{~mL}$ d'eau) (b) pour analyses, dans les proportions de $23 \mathrm{~mL}$ de (a) et de $2 \mathrm{~mL}$ de (b) complétés à $100 \mathrm{~mL}$ avec de l'eau désionisée ou bidistillée. Les benzodiazépines destinées aux étalonnages sont fournies par Euromedex-Lipomed, Mundolsheim, France sous forme d'ampoules titrées à $1 \mathrm{~g} / \mathrm{L}$ dans le méthanol (ou l'acétonitrile pour le 7-aminoflunitrazépam et le

Tableau II : Performances de la technique de référence.

\begin{tabular}{|c|c|c|c|c|c|c|c|c|c|c|}
\hline Benzodiazépine & $\begin{array}{c}\mathbf{N}^{\circ} \\
\text { d'ordre }\end{array}$ & $\begin{array}{l}\text { TR } \\
\text { mn }\end{array}$ & $\begin{array}{c}\text { LDS } \\
\text { mg/L }\end{array}$ & $\begin{array}{c}\text { LBDQ } \\
\mathrm{mg} / \mathrm{L}\end{array}$ & $\begin{array}{c}\text { LHDQ } \\
\mathrm{mg} / \mathrm{L}\end{array}$ & $\begin{array}{l}\mathbf{R} \\
\boldsymbol{\%}\end{array}$ & \multicolumn{2}{|c|}{$\begin{array}{c}\text { Reproductibilité } \\
\text { n=5, CV\% } \\
\text { 0,1mg/L 0,2mg/L }\end{array}$} & \multicolumn{2}{|c|}{$\begin{array}{c}\text { Répétabilité } \\
\text { n=5, CV\% } \\
\text { 0,1mg/L 0,2mg/L }\end{array}$} \\
\hline 7-Aminoflunitrazépam & 1 & 11.03 & 0,01 & 0,03 & 0,30 & 67.0 & 12,9 & 11,3 & 7,7 & 5,1 \\
\hline Bromazépam & 2 & 17.00 & 0,01 & 0,03 & 0,30 & 68.8 & 5,3 & 9,2 & 2,5 & 3,8 \\
\hline Hydroxy-midazolam & 3 & 17.72 & 0,02 & 0,03 & 0,30 & 91.6 & 11,7 & 6,3 & 4,9 & 4,8 \\
\hline Midazolam & 4 & 18.29 & 0,01 & 0,03 & 0,30 & 101.2 & 12,1 & 5,7 & 5,3 & 5,1 \\
\hline Oxazépam & 5 & 21.18 & 0,01 & 0,03 & 0,30 & 101.7 & 8,1 & 4,6 & 4,9 & 2,0 \\
\hline Nitrazépam & 6 & 21.44 & 0,01 & 0,03 & 0,30 & 85.4 & 8,5 & 5,3 & 3,8 & 2,0 \\
\hline Lorazépam & 7 & 22.12 & 0,01 & 0,03 & 0,30 & 81.0 & 3,9 & 2,6 & 2,5 & 2,6 \\
\hline Clonazépam & 8 & 22.90 & 0,01 & 0,03 & 0,30 & 89.8 & 9,6 & 6,4 & 2,3 & 1,4 \\
\hline Estazolam & 9 & 23.47 & 0,02 & 0,03 & 0,30 & 99.6 & 8,6 & 9,3 & 7,1 & 4,3 \\
\hline Desméthyldiazépam & 10 & 23.51 & 0,01 & 0,03 & 0,30 & 101.8 & 7,0 & 5,9 & 3,1 & 2,9 \\
\hline Témazépam & 11 & 24.41 & 0,01 & 0,03 & 0,30 & 100.5 & 10,2 & 7,7 & 5,0 & 3,7 \\
\hline Flunitrazépam & 12 & 24.80 & 0,01 & 0,03 & 0,30 & 91.1 & 8,6 & 6,5 & 2,3 & 1,3 \\
\hline Clobazam & 13 & 25.43 & 0,01 & 0,03 & 0,30 & 92.3 & 10,6 & 5,2 & 4,5 & 4,2 \\
\hline Lormétazépam & 14 & 25.78 & 0,01 & 0,03 & 0,30 & 71.4 & 10,7 & 6,7 & 4,2 & 4,1 \\
\hline Alprazolam & 15 & 25.80 & 0,01 & 0,03 & 0,30 & 99.1 & 7,6 & 7,3 & 7,4 & 5,6 \\
\hline Triazolam & 16 & 26.15 & 0,01 & 0,03 & 0,30 & 99.0 & 4,2 & 2,8 & 2,3 & 1,6 \\
\hline Tétrazépam & 17 & 27.30 & 0,01 & 0,03 & 0,30 & 100.3 & 8,5 & 6,5 & 2,8 & 2,6 \\
\hline Diazépam & 18 & 28.37 & 0,02 & 0,03 & 0,30 & 101.6 & 8,4 & 6,9 & 5,6 & 3,0 \\
\hline Prazépam & 20 & 34.77 & 0,02 & 0,03 & 0,30 & 101.2 & 11,9 & 9,3 & 8,2 & 6,3 \\
\hline Loflazépate d'éthyle (SI) & 19 & 29.13 & & & & & & & & \\
\hline
\end{tabular}

$\mathrm{TR}=$ Temps de rétention, LDS = Limite de Détection, LBDQ = Limite Basse de Quantification, LHDQ = Limite Haute de Quantification, $\mathrm{R}=$ Rendement d'extraction, $\mathrm{CV}=$ Coefficient de variation. 
lorazépam). Des dilutions à $100 \mathrm{mg} / \mathrm{L}$ dans le méthanol ou l'acétonitrile sont adressées aux participants et stockées à $-20^{\circ} \mathrm{C}$. Les solutions mères à $100 \mathrm{mg} / \mathrm{L}$ de benzodiazépines stockées à $-20^{\circ} \mathrm{C}$ se conservent en moyenne une année, les solutions filles à $10 \mathrm{mg} / \mathrm{L}$ se conservent entre 1 et 3 mois selon la molécule.

Les échantillons tests 1 et 2 se présentent sous forme de plasmas lyophilisés surchargés en benzodiazépines en provenance de LGC Promochem, Molsheim, France. Les surcharges théoriques de ces 2 échantillons, inconnues des participants, figurent dans le Tableau III. Les échantillons sont reconstitués avec $2,5 \mathrm{~mL}$ d'eau désionisée ou bidistillée, mélangés par retournements lents et laissés reposés $20 \mathrm{mn}$ à température ambiante. Avant utilisation, ils sont de nouveau mélangés $5 \mathrm{mn}$ mécaniquement par rotation pour assurer une parfaite homogénéité. Après reconstitution, les échantillons sont stables 2 semaines, à l'abri de la lumière et à une température comprise entre +2 et $+8^{\circ} \mathrm{C}$.

Le standard interne, le loflazépate d'éthyle, est fourni aux participants sous forme de produit pur, don de Sanofi-Synthélabo. Une solution mère de standard interne à $100 \mathrm{mg} / \mathrm{L}$ dans le méthanol est préparée et stockée à $-20^{\circ} \mathrm{C}$; une solution fille à $10 \mathrm{mg} / \mathrm{L}$ est réalisée extemporanément au moment de l'étalonnage et de l'évaluation des échantillons tests. La conservation de la solution mère à $100 \mathrm{mg} / \mathrm{L}$ est d'une année, celle de la solution fille à $10 \mathrm{mg} / \mathrm{L}$ de un mois.

\section{Technique d'extraction :}

L'extraction réalisée sur $1 \mathrm{~mL}$ de plasma est effectuée à l'aide de $4 \mathrm{~mL}$ d'un mélange hexane / dichlorométhane $(4 / 3, \mathrm{v} / \mathrm{v})$ en présence de $50 \mu \mathrm{L}$ de loflazépate d'éthyle à $10 \mathrm{mg} / \mathrm{L}$ dans le méthanol et de $2 \mathrm{~mL}$ de tampon carbonate $\mathrm{pH}$ 9,2. Pour l'extraction de certains plasmas lyophilisés reconstitués, le volume d'extraction peut être amené à $6 \mathrm{~mL}$ pour limiter la survenue d'émulsion. Après agitation mécanique à vitesse modérée (10 $\mathrm{min})$ et centrifugation (10 $\mathrm{min})$, la phase organique est prélevée puis évaporée à sec. Le résidu sec est repris par une quantité suffisante de solvant de reprise à optimiser par chaque centre participant selon la technique de détection retenue.

\section{Gammes d'étalonnage :}

Les différentes gammes d'étalonnage correspondant à chaque benzodiazépine sont réalisées au moyen de 6 points de concentrations échelonnés entre 0,03 et $0,30 \mathrm{mg} / \mathrm{L}(0,03,0,05,0,10,0,20,0,25,0,30 \mathrm{mg} / \mathrm{L})$. Les surcharges sont réalisées à partir d'un pool de benzodiazépines à $10 \mathrm{mg} / \mathrm{L}$ préparées à partir des solutions standards à $100 \mathrm{mg} / \mathrm{L}$. Les limites basse et haute de quantification peuvent être adaptées en fonction de la technique de détection utilisée après vérification de l'exactitude et de la fidélité. Pour des techniques de quantification autres que la spectrométrie de masse, certaines benzodiazépines peuvent coéluer et gêner leur quantification mutuelle. Dans ce cas, il convient de prévoir des pools fractionnés de benzodiazépines avant de procéder aux surcharges, ou de modifier le gradient de température en technique CPG ou bien le gradient et la composition de la phase mobile en technique CLHP.

\section{Critères d'acceptabilité :}

L'objectif est de rendre compte au maximum de la dispersion des résultats inter-laboratoires, reflet des différentes conditions de mise en œuvre de la méthode d'extraction, des qualités des étalonnages et du paramétrage de l'appareillage.

Dans ce but, tout résultat inférieur ou égal à $\pm 35 \%$ la valeur théorique est considéré comme acceptable, correspondant à un élargissement des critères d'acceptation fixés par Shah et coll. lors de la conférence de consensus de la FDA (2).

\section{Résultats}

\section{Techniques de séparation et de détection mises en œuvre :}

Les différentes techniques mises en œuvre par les différents participants sont décrites dans le Tableau IV. Aucun des centres n'a utilisé la technique de séparation

Tableau III : Composition des échantillons tests.

\begin{tabular}{|c|c|c|c|}
\hline \multicolumn{2}{|c|}{ Echantillon 1 } & \multicolumn{2}{c|}{ Echantillon 2 } \\
\hline Molécule & Concentration (mg/L) & Molécule & 0,05 \\
\hline diazépam & 0,60 & alprazolam & 0,50 \\
\hline nordiazépam & 0,60 & clobazam & 0,10 \\
\hline lorazépam & 0,10 & midazolam & 0,50 \\
\hline oxazépam & 0,60 & tétrazépam & 0,10 \\
\hline bromazépam & 0,40 & clonazépam & \\
\hline flunitrazépam & 0,05 & & \\
\hline 7- aminoflunitrazépam & 0,05 & & \\
\hline
\end{tabular}




\begin{tabular}{|c|c|c|c|c|c|c|c|c|c|c|}
\hline$=$ & 鹃 & 3 & & 吉 & $\stackrel{\infty}{U}$ & 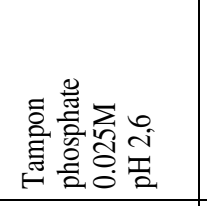 & 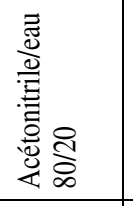 & & 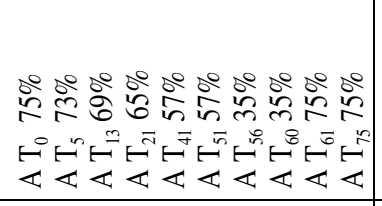 & 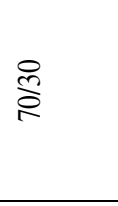 \\
\hline 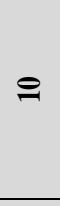 & 善 & $\begin{array}{l}\text { 竞 } \\
1 \\
3 \\
3\end{array}$ & & 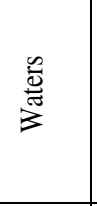 & $\stackrel{\infty}{\circlearrowright}$ & 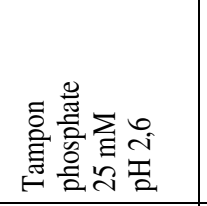 & 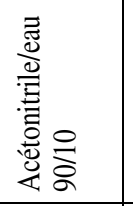 & 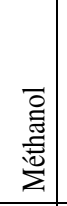 & 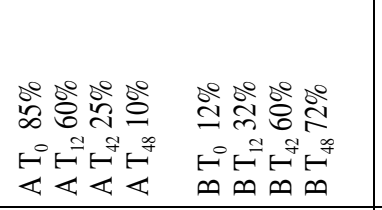 & 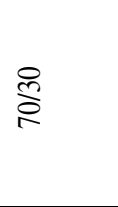 \\
\hline$a$ & 言 & $\begin{array}{l}\text { 竞 } \\
1 \\
3 \\
3\end{array}$ & & 咅 & $\stackrel{\infty}{\Xi}$ & 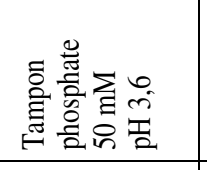 & 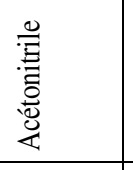 & & 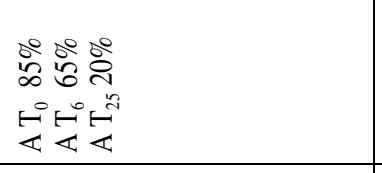 & 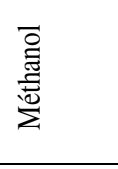 \\
\hline$\infty$ & 害 & $\begin{array}{l}\text { 竞 } \\
1 \\
3 \\
3\end{array}$ & & 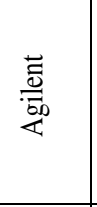 & $\stackrel{\infty}{U}$ & 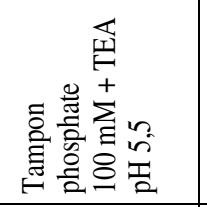 & 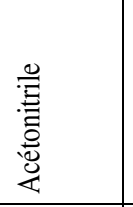 & & 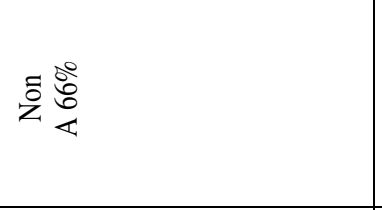 & 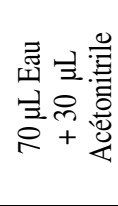 \\
\hline$n$ & 善 & $\begin{array}{l}2 \\
\vdots \\
1 \\
3\end{array}$ & & 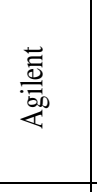 & $\stackrel{\infty}{\circlearrowright}$ & 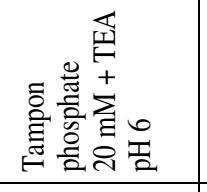 & 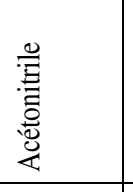 & & 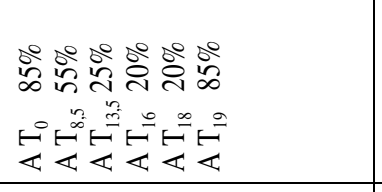 & 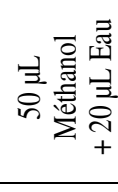 \\
\hline$b$ & 善 & $\begin{array}{l}\text { 音 } \\
1 \\
3 \\
3\end{array}$ & & 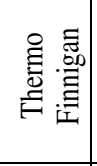 & $\stackrel{\infty}{\Xi}$ & 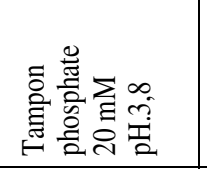 & 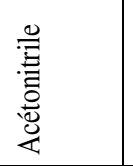 & & 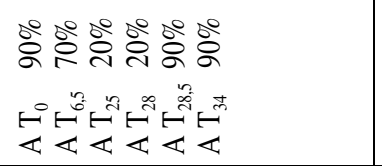 & 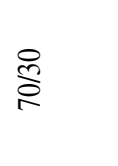 \\
\hline in & 帚 & $\begin{array}{l}\text { 空 } \\
1 \\
3 \\
3\end{array}$ & & $\frac{n}{\bar{m}}$ & $\infty$ & 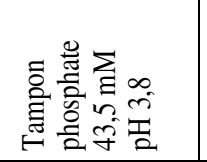 & 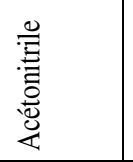 & & 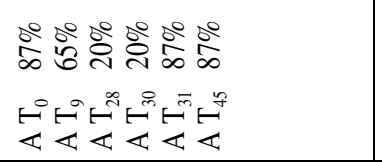 & $\underset{\infty}{\stackrel{m}{*}}$ \\
\hline$\nabla$ & 害 & $\begin{array}{l}\text { 竞 } \\
1 \\
3 \\
3\end{array}$ & & 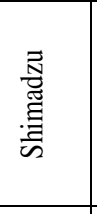 & $\stackrel{\infty}{\Xi}$ & 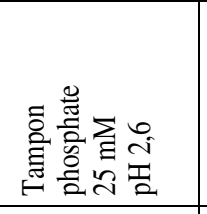 & 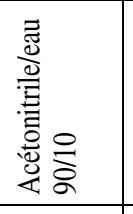 & & 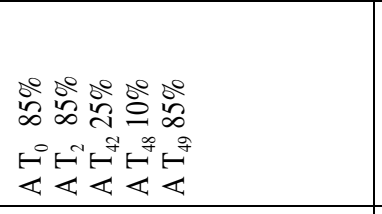 & 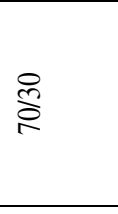 \\
\hline$m$ & 善 & $\sum_{n=1}^{n}$ & $\overline{7}$ & 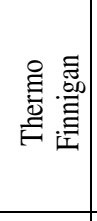 & $\stackrel{\infty}{U}$ & 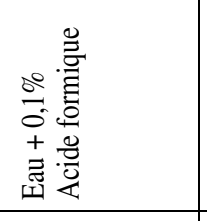 & 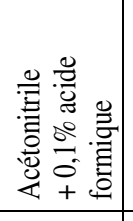 & & 艺造 & 莺 \\
\hline$N$ & 善 & $\sum_{n}$ & 도 & 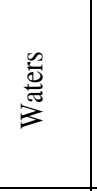 & $\stackrel{\infty}{\circlearrowright}$ & 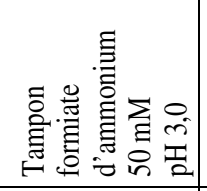 & 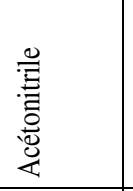 & & 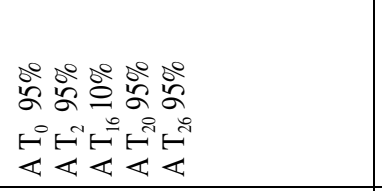 & 号 \\
\hline- & ¿ & $\sum_{n}$ & 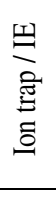 & 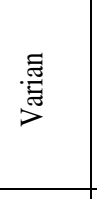 & 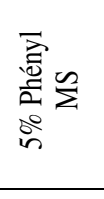 & 帮 & & & 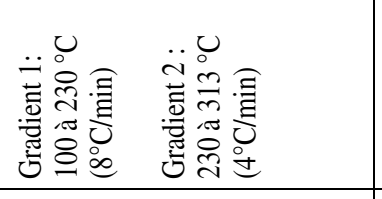 & 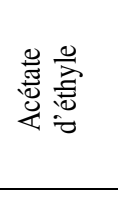 \\
\hline 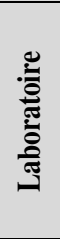 & 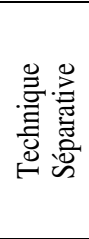 & 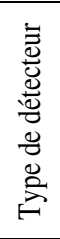 & 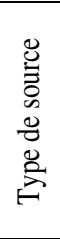 & 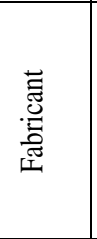 & 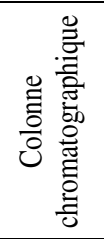 & 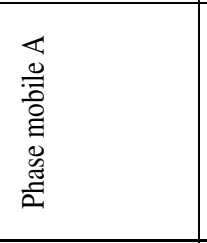 & 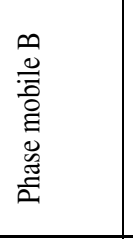 & 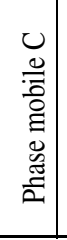 & 壱 & 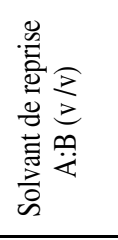 \\
\hline
\end{tabular}


et de détection proposée par défaut. 10 des 11 participants utilisent une technique CLHP, un seul utilise une technique CPG. Parmi les techniques CLHP, 2 sont couplées à un détecteur de masse, 7 à un détecteur à barrette de diodes, et 1 à un détecteur UV. La technique CPG est couplée à un détecteur de masse.

Parmi les 10 techniques CLHP, 9 centres utilisent une colonne séparative de type $\mathrm{C} 18,1$ centre a choisi une colonne C8. Deux techniques CLHP travaillent en mode isocratique. Les techniques de détection UV ou UV- barrette de diodes utilisent un système d'élution variable, constitué d'une solution tampon phosphate de $\mathrm{pH}$ compris entre 2,6 et 6,0 à une concentration variant de 20 à $100 \mathrm{mM}$, d'acétonitrile ou acétonitrile / eau et de méthanol. Pour les techniques CLHP associées à la spectrométrie de masse, l'une utilise un tampon formiate d'ammonium $50 \mathrm{mM}$ à $\mathrm{pH} \mathrm{3,0}$ (phase $\mathrm{A}$ ) et de l'acétonitrile (phase B), l'autre utilise un mélange eau avec $0,1 \%$ d'acide formique (phase $\mathrm{A}$ ) et un mélange acétonitrile avec $0,1 \%$ d'acide formique (phase B). La technique CPG utilise une colonne 5\% phényl SM en gradient de température.

\section{Résultats :}

Le Tableau V fait état des résultats de l'étude. La figure 1 représente les histogrammes de répartition des résultats rendus, molécule par molécule, ainsi que leurs limites d'acceptabilité. Sur les 132 résultats attendus, 125 résultats ont été communiqués parmi lesquels 101 ont satisfait aux critères d'admissibilité précédemment définis, représentant $80,8 \%$ des résultats rendus, ou 76,5\% des résultats attendus, réduisant de 11 à 7 , selon la molécule, le nombre de participants ayant rendu des résultats satisfaisants. Si la limite d'admissibilité avait été fixée à $\pm 15 \%$ la valeur théorique selon les recommandations de Shah et coll. (2), seulement $59 \%$ des résultats auraient été satisfaisants.

Globalement, dans les conditions d'admissibilité défi- nies dans l'étude, 7 résultats manquants et 24 résultats exclus ont été enregistrés. 4 laboratoires ont rendu $100 \%$ de résultats acceptables, 2 ont rendu 1 résultat non acceptable, 1 a rendu 1 résultat non acceptable et 1 résultat manquant, 1 a rendu 2 résultats manquants, 1 a rendu 4 résultats non acceptables et 1 résultat manquant, 1 a rendu 6 résultats non acceptables et 3 résultats manquants, 1 a rendu 11 résultats non acceptables.

\section{Discussion}

Les 7 résultats manquants s'expliquent par 5 interférences (répartis sur 4 sites) et 2 par défaut de sensibilité. En ce qui concerne les interférences observées, elles concernent la détermination du flunitrazépam pour 2 laboratoires, du tétrazépam et du midazolam pour 1 laboratoire, du lorazépam pour 1 laboratoire. On peut penser qu'un délai plus long accordé à l'étude aurait permis de modifier les conditions expérimentales des 4 laboratoires concernés et de régler le problème essentiellement lié à un mauvais paramétrage des conditions chromatographiques. Les 2 résultats manquants ont été enregistrés par le laboratoire utilisant une technique CPG. 25 des 31 résultats manquants ou exclus se répartissent sur 3 sites, dont 20 sur 2 sites : il s'agit d'une part du laboratoire utilisant la technique CLHP couplée à un détecteur UV (1 interférence, 10 exclusions pour résultats hors limites d'admissibilité) manquant d'expérience pour le dosage des benzodiazépines, d'autre part le site utilisant la technique CG-SM-IE (2 défauts de sensibilité, 7 exclusions pour résultats hors limites d'admissibilité) pour lequel le loflazépate d'éthyle s'est révélé être un standard interne mal adapté, avec des sensibilités médiocres pour le 7-aminoflunitrazépam et le clonazépam. Le troisième site (2 interférences, 3 exclusions pour résultats hors limites d'admissibilité) a été particulièrement gêné par des problèmes d'émulsion liés à la nature lyophilisée des échantillons tests.

Tableau V:Résultats de l'étude.

\begin{tabular}{|c|c|c|c|c|c|c|c|}
\hline Molécule & $\begin{array}{c}\mathrm{Nb} \\
\text { participants }\end{array}$ & $\begin{array}{l}\text { Nb résultats } \\
\text { inclus }\end{array}$ & $\begin{array}{c}\text { Surcharge } \\
\text { Théorique } \\
\text { mg/L }\end{array}$ & $\begin{array}{c}\text { Moyenne } \\
\text { Résultats } \\
\text { mg/L }\end{array}$ & $\begin{array}{c}\text { Exactitude } \\
\%\end{array}$ & $\begin{array}{l}\text { Déviation } \\
\text { standard }\end{array}$ & $\begin{array}{c}\text { Coeff. } \\
\text { variation }\end{array}$ \\
\hline diazépam & 11 & $9(2 \mathrm{ex})$ & 0,600 & 0,648 & 108,0 & 101,6 & 15,6 \\
\hline nordiazépam & 11 & $9(2 \mathrm{ex})$ & 0,600 & 0,585 & 97,6 & 99,4 & 16,9 \\
\hline lorazépam & 11 & 8 (2ex, lint) & 0,100 & 0,112 & 112,2 & 8,8 & 7,8 \\
\hline oxazépam & 11 & $7(4 \mathrm{ex})$ & 0,600 & 0,705 & 117,5 & 73,1 & 10,3 \\
\hline bromazépam & 11 & $8(3 \mathrm{ex})$ & 0,400 & 0,401 & 100,3 & 67,4 & 16,7 \\
\hline flunitrazépam & 11 & 7 (2ex,2int) & 0,050 & 0,057 & 115,6 & 13,2 & 22,8 \\
\hline 7-aminoflunitrazépam & 11 & 8 (2ex,1ds) & 0,050 & 0,051 & 103,2 & 12,8 & 24,9 \\
\hline alprazolam & 11 & $8(3 \mathrm{ex})$ & 0,050 & 0,047 & 94,4 & 5,6 & 11,9 \\
\hline clobazam & 11 & 11 & 0,500 & 0,463 & 92,6 & 79,9 & 17,2 \\
\hline midazolam & 11 & 9 (1ex, lint) & 0,100 & 0,106 & 106,8 & 23,4 & 21,9 \\
\hline tétrazépam & 11 & 8 (2ex, lint) & 0,500 & 0,423 & 84,7 & 63,9 & 15,0 \\
\hline clonazépam & 11 & 9 (1ex,1ds) & 0,100 & 0,095 & 95,0 & 12,4 & 13,1 \\
\hline
\end{tabular}

ex $=$ exclusion pour résultats hors limites d'admissibilité - int = interférence - ds = défaut de sensibilité 

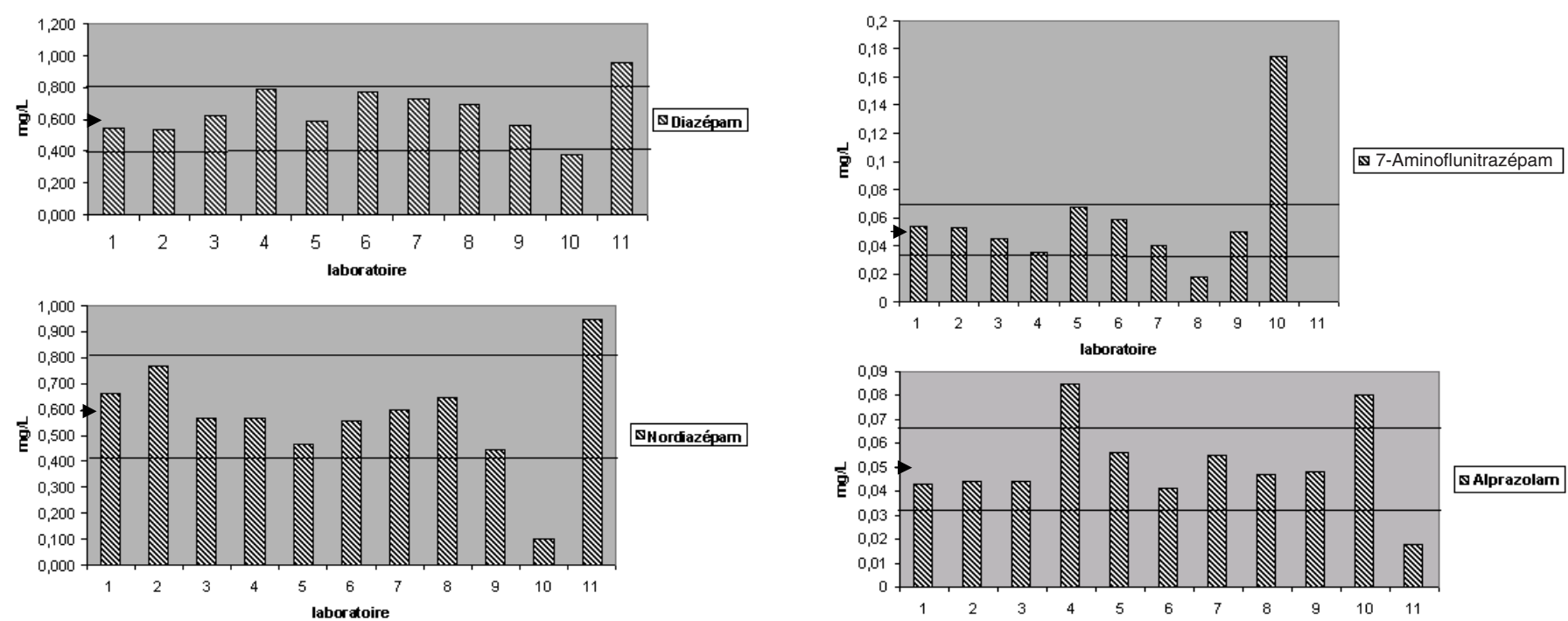

SHordazépam

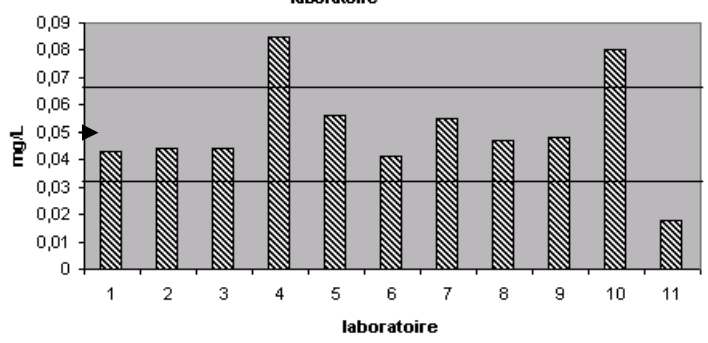

\$ Alprazolam
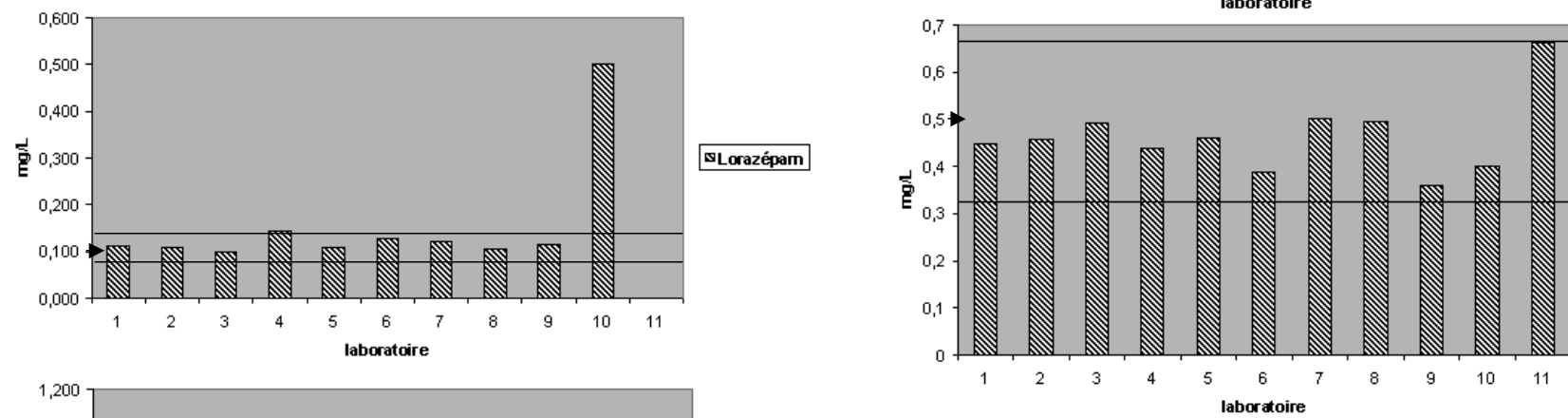

\$Clobazam

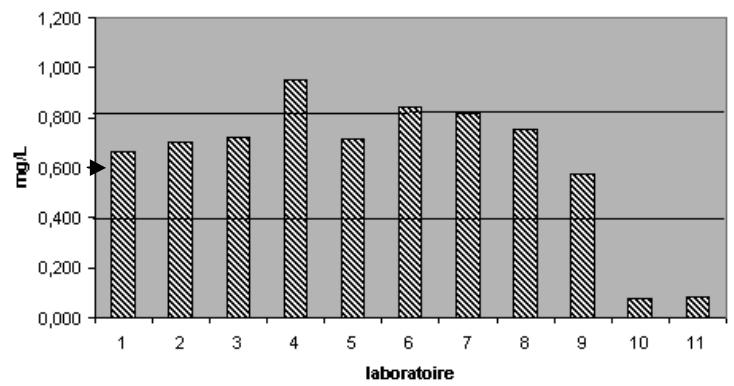

\$0xazépam

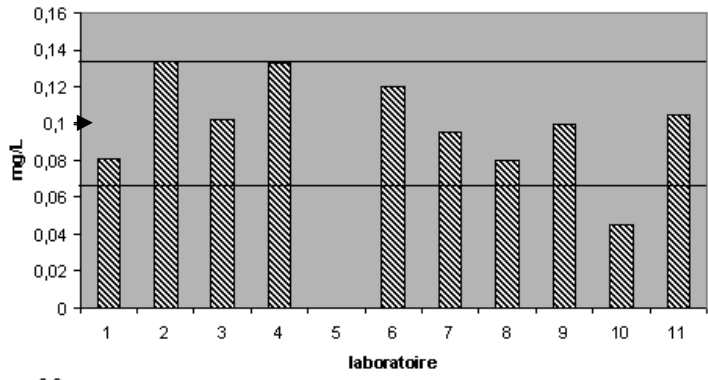

S Mdazolam
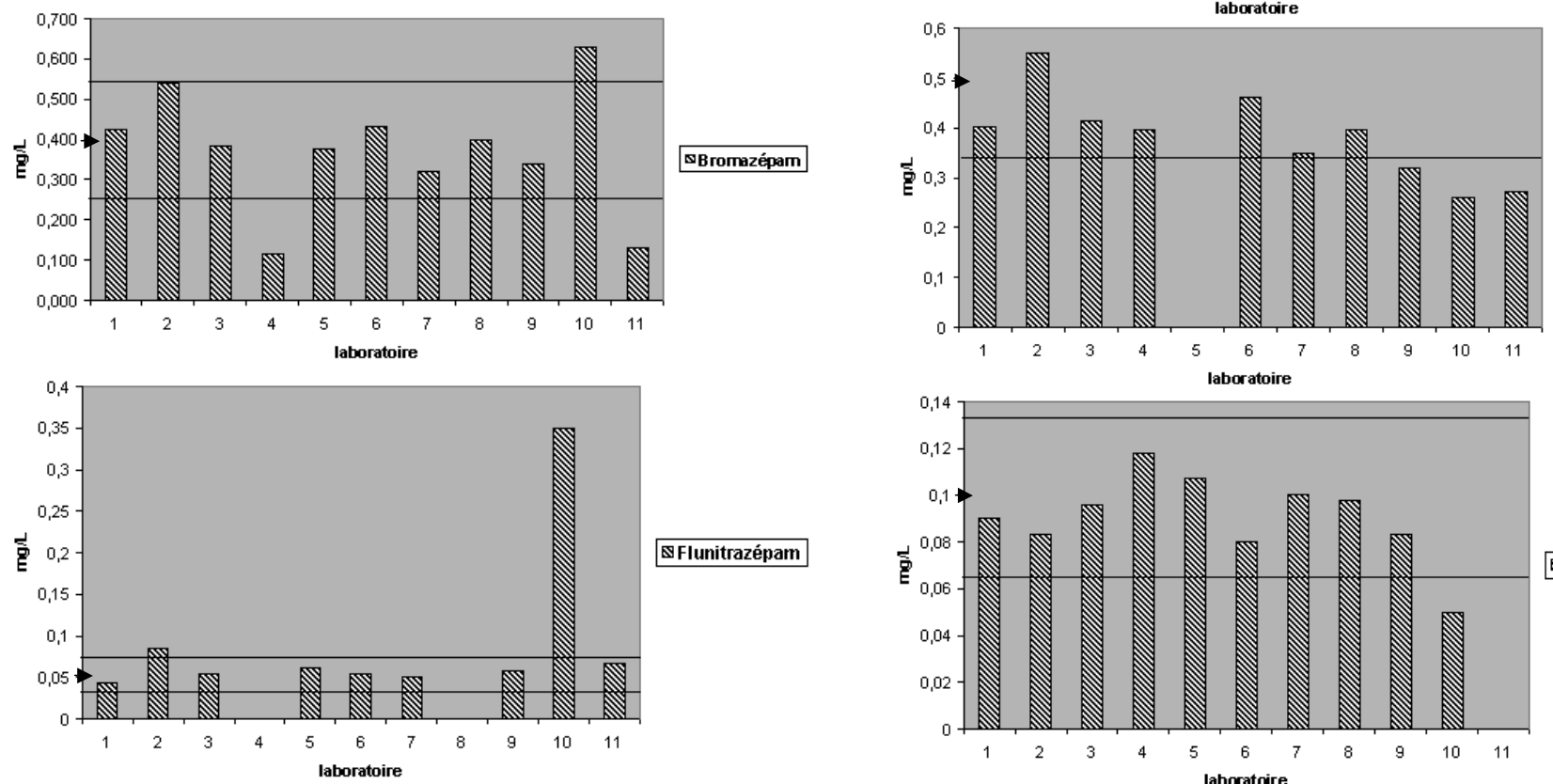

\$ Tétrazépam

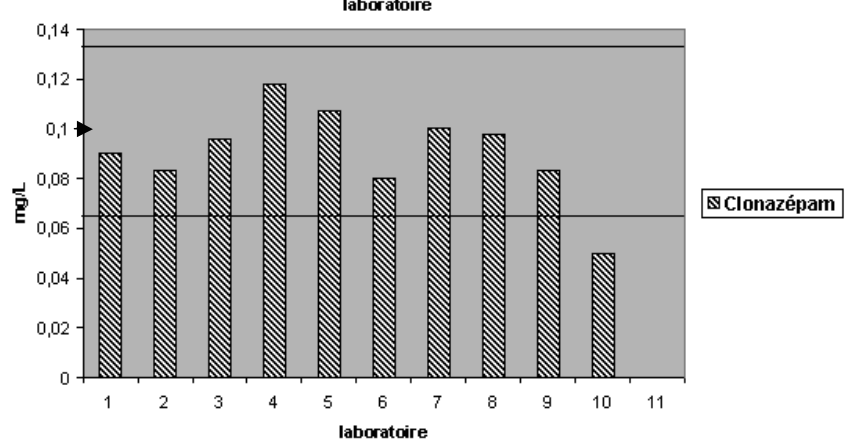

Figure 1 : Représentation graphique des résultats, valeurs cibles $(\rightarrow)$ et limites d'acceptabilité (-). Limite d'acceptation $\leq \pm 35 \%$ la valeur théorique. 
Ce risque avait été signalé en début d'étude, plus souvent rencontré lors de l'extraction de plasmas lyophilisés reconstitués. Dans ce cas précis, il aurait été souhaitable de revoir les conditions d'agitation soumises aux échantillons. Si l'utilisation du dichlorométhane seul comme solvant d'extraction des benzodiazépines diminue le risque d'émulsion, le choix du mélange hexane / dichlorométhane se justifie par l'augmentation des rendements d'extractions des produits de métabolisation hydroxylés des benzodiazépines. Ainsi, le rapport $4: 3 \mathrm{v} / \mathrm{v}$ du mélange hexane / dichlorométhane retenu par le laboratoire coordonnateur a permis d'atteindre des rendements d'extraction optimisés pour les benzodiazépines hydroxylées (oxazépam, lorazépam, lormétazépam, témazépam) compris entre $71,4 \%$ pour le lormétazépam et 101,7\% pour l'oxazépam (Tableau II). En ce qui concerne les résultats exclus de l'étude, ils touchent plus particulièrement l'oxazépam (4 sites), le bromazépam et l'alprazolam (3 sites). Inversement, tous les participants ont fourni un résultat acceptable pour le clobazam. La moindre dispersion des résultats concerne le lorazépam (CV 7,8\%) dont le rendement d'extraction est de $81 \%$ avec de faibles coefficients de variation des résultats de validation (Tableau II). La plus grande dispersion concerne le 7-aminoflunitrazépam (CV 24,9\%) dont le rendement d'extraction est le plus faible, avec des coefficients de variation élevés pour les résultats de validation (Tableau II). L'erreur calculée sur la mesure (exactitude) est comprise entre $84,7 \%$ pour le tétrazépam et $117,5 \%$ pour l'oxazépam, valeurs proches de l'erreur d'exactitude maximale admise par Shah et col. (2), fixée à $\pm 15 \%$ la valeur théorique. Dans l'ensemble, la technique d'extraction proposée a donc permis d'obtenir $100 \%$ de résultats satisfaisants lorsqu'elle a été couplée à une technique d'analyse CLHP dans des conditions expérimentales préalablement testées par le laboratoire participant. Bien qu'aucune étude comparative n'ait été mise en œuvre entre la technique d'extraction usuelle des benzodiazépines pratiquée par les différents sites et la technique testée, il ressort des participants à l'étude les informations suivantes : la technique est unanimement reconnue très simple d'utilisation, elle utilise des solvants différents de ceux utilisés par les participants (éther ou dichlorométhane en milieu alcalin, mélange éther / hexane / dichlorométhane / alcool isoamylique notamment) tout en conduisant à des résultats voisins, confirmant les conclusions de Drummer (3), à savoir que sur plus de 20 solvants utilisés seuls ou en association parmi lesquels l'éther, le toluène, le dichlorométhane, le chloroforme, le chlorure de butyle et l'hexane, peu de différences séparent les résultats et les auteurs aboutissent tous à des rendements supérieurs à $60 \%$ pour la plupart des benzodiazépines, les extractions se conduisant en milieu légèrement alcalin, voire sans addition de tampon. Par ailleurs, la technique utilise le loflazépate d'éthyle comme standard interne, benzodiazépine peu prescrite, alors que d'autres benzodiazépines sont utilisées par les participants, notamment le prazépam. Enfin, un laboratoire participant a depuis adopté la technique en pratique courante.

\section{Conclusion}

L'étude confirme que l'extraction proposée est robuste et simple d'utilisation, accessible à tous les laboratoires appelés à analyser les benzodiazépines par CLHP dans le plasma. En pratique, la technique d'extraction a pu être testée avec succès par 8 des 11 participants à l'étude : 4 laboratoires ont rendu un sans faute, 4 autres ont eu 1 ou 2 résultats exclus ou gênés par une interférence. Enfin, les 3 autres participants ont rencontré des difficultés majeures : 1 site par la survenue d'émulsions lors du traitement des échantillons tests lyophilisés, 1 site par manque de temps et d'expérience, le troisième mettant en cause le choix du standard interne en technique CPG, le loflazépate d'éthyle n'ayant pas fait l'objet d'une étude préalable par cette technique.

\section{Références}

1. Nicolas O., Farenc C., Bressolle F. Stratégie de validation de méthodes de dosage en bioanalyse en vue d'études pharmacocinétiques et toxicologiques. Ann.Toxicol. Anal. 2004 ; XVI (2) : 118 -127.

2. Shah V. P., Midha K. K., Findlay J.W.A., Hill H.M., Hulse J. D., McGilveray I.J., McKay G., Miller K.J., Patnaik R. N., Powell M. L., Tonelli A., Viswanathan C. T., Yacobi A. Bioanalytical method validation - A revisit with a decade of progress. Pharm. Res. 2000 ; 17 : 1551-7.

3. Drummer. O. H. Methods for measurement of benzodiazepines in biological samples. J. Chromatrogr. B. 1998 ; $713: 3-25$. 